5. Chai LY, et al. Anti-Aspergillus human host defence relies on type $1 \mathrm{~T}$ helper (Th1), rather than type 17 T helper (Th17), cellular immunity. Immunology. 2010;130(1):46-54.

6. Cramer RA, Rivera A, Hohl TM. Immune responses against Aspergillus fumigatus: what have we learned? Curr Opin Infect Dis. 2011;24(4):315-322.

7. Kreindler JL, et al. Vitamin D3 attenuates Th2 responses to Aspergillus fumigatus mounted by CD4 $\mathrm{T}$ cells from cystic fibrosis patients with allergic bronchopulmonary aspergillosis. J Clin Invest. 2010;120(9):3242-3254.

8. Tramsen L, et al. Clinical-scale generation of human anti-Aspergillus $\mathrm{T}$ cells for adoptive immunotherapy. Bone Marrow Transplant. 2009;43(1):13-19.

9. Cenci E, Mencacci A, Bacci A, Bistoni F, Kurup VP, Romani L. T cell vaccination in mice with invasive pulmonary aspergillosis. J Immunol. 2000;165(1):381-388

10. Hohl TM, et al. Inflammatory monocytes facilitate adaptive CD4 $\mathrm{T}$ cell responses during respiratory fungal infection. Cell Host Microbe. 2009;6(5):470-481.

11. Tao J, et al. Aspergillus fumigatus extract differentially regulates antigen-specific $\mathrm{CD}^{+}$and $\mathrm{CD}^{+} \mathrm{T}$ cell responses to promote host immunity. J Leukoc Biol. 2006;80(3):529-537.

12. Carvalho A, et al. TLR3 essentially promotes protective class I-restricted memory $\mathrm{CD}^{+} \mathrm{T}$-cell responses to Aspergillus fumigatus in hematopoietic transplanted patients. Blood. 2012;119(4):967-977.

13. De Luca A, et al. CD4 ${ }^{+} \mathrm{T}$ cell vaccination overcomes defective cross-presentation of fungal antigens in a mouse model of chronic granulomatous disease. J Clin Invest. 2012;122(5):1816-1831.

14. Schnorrer $\mathrm{P}$, et al. The dominant role of $\mathrm{CD}^{+}$ dendritic cells in cross-presentation is not dictated by antigen capture. Proc Natl Acad Sci U S A. 2006;103(28):10729-10734.

15. Chemali M, Radtke K, Desjardins M, English L. Alternative pathways for MHC class I presenta- tion: a new function for autophagy. Cell Mol Life Sci. 2011;68(9):1533-1541.

16. Kurts C, Robinson BW, Knolle PA. Cross-priming in health and disease. Nat Rev Immunol. 2010;10(6):403-414.

17. Donaldson $\mathrm{M}$, et al. p47phox-deficient immune microenvironment signals dysregulate naive T-cell apoptosis. Cell Death Differ. 2009;16(1):125-138

18. Crotzer VL, Blum JS. Autophagy and adaptive immunity. Immunology. 2010;131(1):9-17.

19. Hasui M, et al. Decreased CD4+CD29+ (memory T) cells in patients with chronic granulomatous disease. J Infect Dis. 1993;167(4):983-985.

20. Araki K, et al. mTOR regulates memory CD8 T-cell differentiation. Nature. 2009;460(7251):108-112.

21. Jagannath C, Lindsey DR, Dhandayuthapani S, Xu Y, Hunter RL Jr, Eissa NT. Autophagy enhances the efficacy of BCG vaccine by increasing peptide presentation in mouse dendritic cells. Nat Med. 2009;15(3):267-276.

\title{
Enhancing immune responses to limit chronic immune activation during SIV
}

\author{
Jacob D. Estes \\ AIDS and Cancer Virus Program, SAIC-Frederick Inc., Frederick National Laboratory for Cancer Research, Frederick, Maryland, USA.
}

\begin{abstract}
The persistent immune activation that is typical of HIV-1 and SIV infection results in exhaustion and dysfunction of $T$ and $B$ cells; in $T$ cells, this is marked by increased expression and signaling through the inhibitory receptor programmed death-1 (PD-1). Targeting this exhaustion pathway could result in improved antiviral immune responses, but there have been concerns that it would also lead to increased inflammation and immunopathology. In this issue of the JCI, Dyavar Shetty et al. demonstrate that blocking PD-1 actually reduced proinflammatory responses and improved immunity in the gut of SIV-infected rhesus macaques, suggesting that this might have therapeutic potential to prevent opportunistic infections in HIV-infected patients.
\end{abstract}

Persistent immune activation is a hallmark of HIV-1 infection in humans and pathogenic SIV infection in rhesus macaques (RMs). HIV and SIV infections induce robust, generalized inflammatory responses that begin during acute infection and lead to pathological systemic immune activation, marked by microbial translocation, fibrotic damage of lymphoid tissues, and $\mathrm{CD}^{+}{ }^{+} \mathrm{T}$ cell loss, processes that contribute to pathogenesis and disease progression. Pathological chronic infections with primate immunodeficiency viruses are further characterized by exhausted and dysfunctional $\mathrm{T}$ and $\mathrm{B}$

Conflict of interest: The author has declared that no conflict of interest exists.

Citation for this article: J Clin Invest. 2012; 122(5):1611-1614. doi:10.1172/JCI63389. cell antiviral immune responses $(1,2)$ that contribute to the inability of the host to eliminate the persistent viral pathogen. The exhaustion of virus-specific $\mathrm{T}$ cells in chronically persistent virus infections is not unique to lentivirus infections, as this phenomenon was first demonstrated in the murine lymphocytic choriomeningitis virus (LCMV) model $(3,4)$, and subsequently virus-specific $\mathrm{T}$ cell exhaustion was demonstrated in HIV, HBV, and HCV infections of humans (5-8) and SIV infection of RMs $(9,10)$.

\section{Can preventing exhaustion improve immune responses?}

Exhausted $\mathrm{T}$ cells are characterized by increased expression of the inhibitory receptor programmed death-1 (PD-1), and in the LCMV model, blockade of the
PD-1 pathway restored virus-specific $T$ cell function, leading to viral clearance (11). While blockade of PD-1 enhances HIV- and SIV-specific T cell cytokine production and proliferation in vitro $(9,10)$, initially there was skepticism that this method would restore immunological function in the pathogenic RM SIV model in vivo (12). This skepticism was based on the premise that if in vivo blockade of the PD-1 inhibitory receptor in chronic SIV infection could result in enhanced antiviral $\mathrm{T}$ and $\mathrm{B}$ cell responses, it could also potentially exacerbate bystander $\mathrm{T}$ cell activation in an already persistent inflammatory setting, potentially leading to accelerated disease progression.

Studies using anti-PD-1 antibody in chronically SIV-infected RMs have produced mixed results. Finnefrock et al. showed that a single injection of anti-PD1 antibody produced no change in SIVspecific $T$ cell function or numbers, with a transient increase in viral loads (13), while Velu et al. demonstrated that four repeated administrations of anti-PD-1 antibody resulted in enhanced SIV-specific immunity and prolonged survival (14). In this issue of the JCI, this latter group expands their analysis of anti-PD-1-treated RMs in an attempt to understand the putative mechanism for prolonged survival (15). 

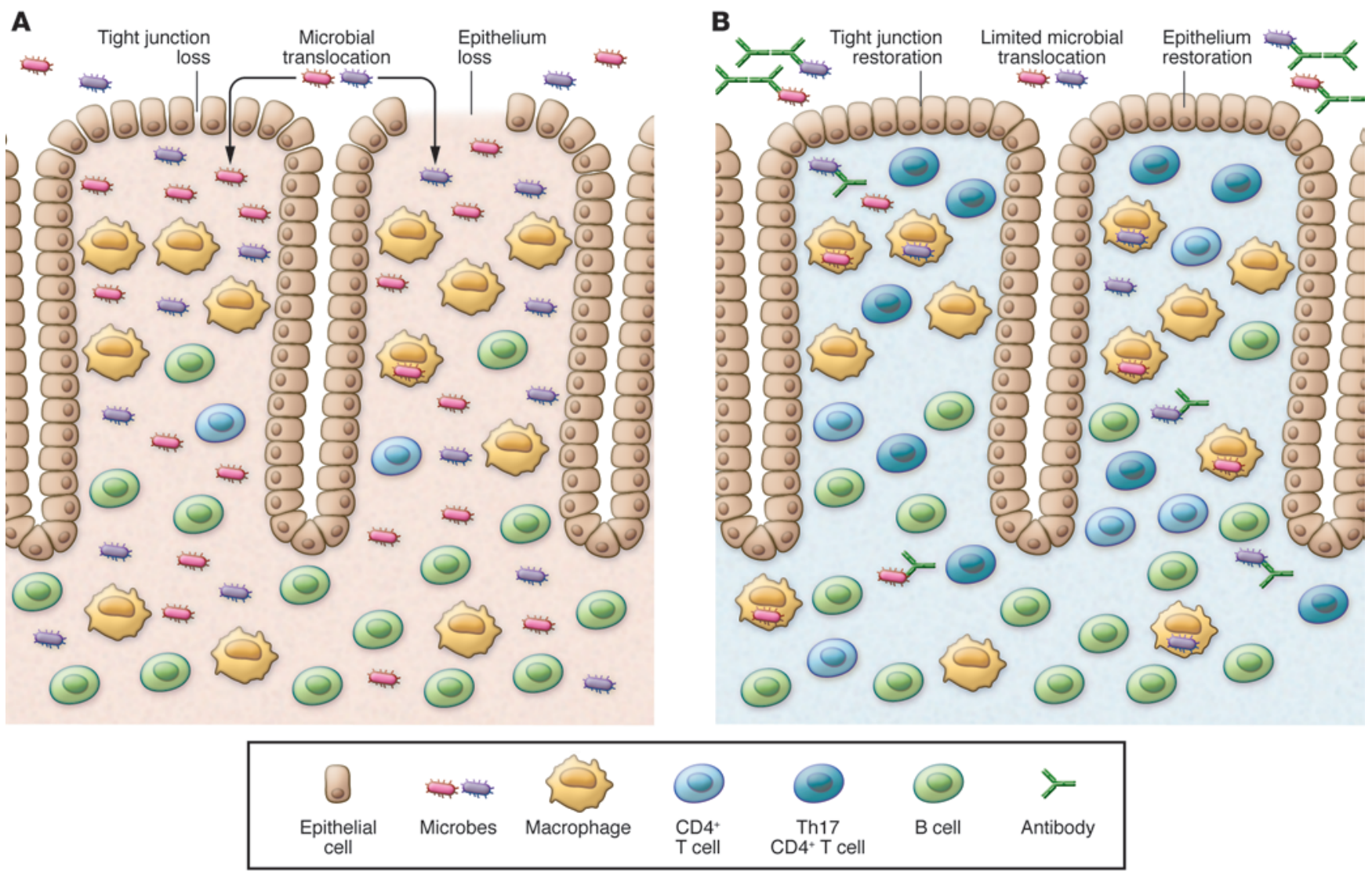

\section{Figure 1}

Simplified model of intestinal immunopathology and restoration with anti-PD-1 antibody treatment in SIV infection. (A) SIV infection results in a chronic proinflammatory environment, leading to exhausted/dysfunctional T and B cells, increased epithelial turnover, multifocal epithelial breaches and loss, microbial translocation, dramatically elevated ISGs, and macrophage dysfunction including inadequate microbial constituent clearance. (B) Treatment of SIV-infected RMs with anti-PD-1 antibody results in a reduced proinflammatory environment, significantly increased SIV-specific and microbial T and B cell function, increased epithelial barrier maintenance and function with increased tight junction expression, reduced microbial translocation, reduced ISG expression, and potentially restored macrophage function including improved microbial constituent clearance. However, the mechanisms that underlie the effects of anti-PD-1 antibody action are incompletely understood.

They demonstrate that although anti-PD1 antibody treatment in SIV-infected RMs was initially intended to enhance $T$ and $B$ cell SIV responses as a means of viral control, it resulted in a significant reduction in proinflammatory responses, leading to enhanced gut immunity, reduced microbial translocation, restoration of the integrity of the gastrointestinal (GI) epithelial barrier, limited opportunistic infections, and significantly prolonged survival compared with controls (Figure 1).

That anti-PD-1 antibody treatment would result in reduced proinflammatory responses rather than enhancing $\mathrm{T}$ and $\mathrm{B}$ cell effector functions and proliferative capacity seems somewhat paradoxical. Nevertheless, anti-PD-1 antibody treatment of chronically SIV-infected RMs resulted in significant downregulation of IFN stimulatory genes (ISGs) (15), which are elevated in association with chronic immune activation and correlate with faster disease progression in SIV-infected RMs $(16,17)$.

\section{Gut reaction to viral infection}

The reduction in ISGs becomes more understandable when seen in the context of our current understanding of the pathogenesis of immune activation in pathogenic SIV infection. Anti-PD-1 treatment resulted in a reduced level of microbial translocation, demonstrated by reduced plasma LPS levels. The effect of anti-PD-1 antibody treatment on ISG expression and microbial translocation was independent of gut viral burden, as anti-PD-1-treated and control RMs had equivalent levels of SIV viral RNA in colonic tissue samples (15). Strong downregulation of type I IFN responses with anti-PD-1 antibody treat- ment in the absence of any effect on gut viral burden reinforces the hypothesis that SIV infection disrupts the GI epithelial barrier, resulting in microbial translocation that in turn helps drive immune activation (18). GI epithelial barrier damage and associated microbial translocation are spatially associated with type I IFN expression within the lamina propria and lymphoid tissues of chronically SIV-infected RMs in a manner not strictly linked to local viral replication, supporting the hypothesis that microbial products - rather than local viral replication - stimulate innate inflammatory responses within the damaged gut (18).

Intriguingly, Dyavar Shetty and colleagues found that anti-PD-1 antibody treatment appeared to help restore GI epithelial barrier integrity, resulting in increased expression in the gut tissue of 
genes associated with tight junctions, along with reduced microbial translocation and type I IFN responses both locally and systemically (15). It is unclear whether the effect on GI epithelial cells in anti-PD-1 antibody-treated animals is due to direct effects on their survival (as these cells do express the PD-L1 ligand) or indirect effects from reduced inflammation and ISG expression due to better clearance of microbial constituents and restoration of the GI epithelial barrier. Regardless of the mechanism, anti-PD-1 antibody treatment significantly reduced microbial translocation and associated ISG expression, changes that correlated with improved survival for anti-PD-1treated animals (15).

\section{Making connections}

While the effect of anti-PD-1 antibody treatment on GI epithelial cell integrity may have been unexpected, during chronic lentiviral infections the exhaustion of $\mathrm{T}$ and $B$ cells may be partially independent of antigenic specificity, reflecting instead the cumulative effects of the overall local inflammatory milieu in tissue sites. Thus, one potential factor contributing to sustained microbial translocation in chronic HIV and SIV infections may be exhaustion of microbe-specific $\mathrm{T}$ and $\mathrm{B}$ cells within the gut and dysfunctions in resident gut macrophages, collectively limiting microbial clearance. Thus, anti-PD-1 antibody treatment may improve not only SIV-specific $\mathrm{T}$ and $\mathrm{B}$ cell responses, but also $\mathrm{T}$ and $\mathrm{B}$ cell responses to common gut-resident bacteria. Indeed, Dyavar Shetty et al. found that anti-PD-1 antibody treatment had an important effect on adaptive immune responses to common gut-resident bacteria (15). Specifically, treatment significantly increased serum Campylobacter-specific antibody levels and Campylobacter-specific and Salmonella-specific peripheral blood $\mathrm{CD}^{+} \mathrm{T}$ cell responses in most of the anti-PD-1-treated RMs. In contrast, in control RMs the $\mathrm{CD}^{+} \mathrm{T}$ cell responses to these same gut-resident bacteria decreased over time, despite ongoing exposure to gut microbes, as reflected in evidence of microbial translocation (15).

In addition to the direct impact of anti-PD-1 treatment on restoring $\mathrm{T}$ and $B$ cell function, several recent studies have implicated PD- 1 expression and ligation on macrophages in either the development of macrophage dysfunction or $\mathrm{CD}^{+} \mathrm{T}$ cell dysfunction (19,
20), suggesting another mechanism by which the immunoregulatory effects of PD-1 expression extend beyond direct PD-1-mediated $T$ and $B$ cell exhaustion. Because a recent study suggested that there may be a dysfunction in the ability of lamina propria macrophages to effectively phagocytose translocated microbial constituents in SIV-infected RMs (18), it will be important to determine whether anti-PD-1 antibody treatment results in improved function and microbial clearance by gut macrophages.

Since HIV infection is associated with exhausted/dysfunctional $\mathrm{T}$ and $\mathrm{B}$ cell responses to the virus, and presumably to other pathogens, an important treatment outcome in HIV patient care would be improved immune responses to these pathogens, particularly the etiologic agents of opportunistic infections. However, it is important to note that deletion of pathogen-specific $T$ cells and not just their dysfunction can contribute to the development of opportunistic infections (21). Dyavar Shetty et al. observed a marked reduction in putative opportunistic infections in anti-PD-1 antibodytreated chronically SIV-infected RMs despite continued high levels of viral replication (15), suggesting that even in the absence of antiretroviral therapy, inhibiting the PD-1 signaling pathway may be able to overcome $\mathrm{T}$ and $\mathrm{B}$ cell exhaustion/dysfunction and thereby improve clinical outcome independent of effects on viral load or $\mathrm{CD}^{+} \mathrm{T}$ cell count (14), two commonly used clinical indicators of disease progression (21).

\section{Perspectives}

While the results reported by Dyavar Shetty and colleagues are provocative and encouraging, their study leaves many important questions unanswered. For example, is the effect of anti-PD-1 antibody treatment sufficient to confer a phenotype similar to that seen in non-pathogenic, non-progressive SIV infections? What effect does anti-PD-1 antibody treatment have on generalized $\mathrm{T}$ and $\mathrm{B}$ cell activation and proliferation during pathogenic HIV and SIV infections in the absence of attenuated viral replication? What global effect does antiPD-1 treatment have on the cytokine milieu within lymphoid and gut tissue compartments, and how does this impact tissue architecture regeneration? What impact does anti-PD-1 treatment have on macrophage survival and function, particularly within the GI tract? Would longer anti-PD-1 antibody treatment or additional treatment cycles result in sustained reductions in microbial translocation, low levels of ISGs, and improved GI tract $\mathrm{CD}^{+} \mathrm{T}$ cell (particularly Th17 cell) recovery? Does blockade of $\mathrm{PD}-1$ signaling in GI tract epithelial cells directly result in improved enterocyte function and regenerative capacity?

Though these and other important questions will need to be addressed, the unanticipated benefits following antiPD1 treatment underscore the complexity of the immunopathology of AIDS virus infections and provide support for considering evaluation of anti-PD-1 antibody treatment in small, targeted human clinical trials aimed at decreasing immune activation and enhancing viral specific immunity.

\section{Acknowledgments}

I would like to thank Jeffrey Lifson and Brandon Keele for their careful reading of and advice on this commentary. This work was funded with federal funds from the National Cancer Institute, NIH, under contract no. HHSN261200800001E. The content of this publication does not necessarily reflect the views or policies of the Department of Health and Human Services, nor does mention of trade names, commercial products, or organizations imply endorsement by the US Government.

Address correspondence to: Jacob D. Estes, NCI/SAIC Frederick Inc., AIDS and Cancer Virus Program, Frederick, Maryland 21702, USA. Phone: 301.846.7641; Fax: 301.846.5588; E-mail: estesj@mail.nih.gov.

1. El-Far M, et al. T-cell exhaustion in HIV infection. Curr HIV/AIDS Rep. 2008;5(1):13-19.

2. Moir S, Fauci AS. Pathogenic mechanisms of Blymphocyte dysfunction in HIV disease. J Allergy Clin Immunol. 2008;122(1):12-19.

3. Zajac AJ, et al. Viral immune evasion due to persistence of activated $T$ cells without effector function. J Exp Med. 1998;188(12):2205-2213.

4. Gallimore A, et al. Induction and exhaustion of lymphocytic choriomeningitis virus-specific cytotoxic T lymphocytes visualized using soluble tetrameric major histocompatibility complex class I-peptide complexes. J Exp Med. 1998;187(9):1383-1393.

5. Day CL, et al. PD-1 expression on HIV-specific T cells is associated with T-cell exhaustion and disease progression. Nature. 2006;443(7109):350-354.

6. Trautmann L, et al. Upregulation of PD-1 expression on HIV-specific CD8+ T cells leads to reversible immune dysfunction. Nat Med. 2006; 12(10):1198-1202.

7. Petrovas C, et al. PD-1 is a regulator of virus-specific CD8+ T cell survival in HIV infection. J Exp 
Med. 2006;203(10):2281-2292.

8. Rehermann B, Nascimbeni M. Immunology of hepatitis B virus and hepatitis $C$ virus infection. Nat Rev Immunol. 2005;5(3):215-229.

9. Velu V, et al. Elevated expression levels of inhibitory receptor programmed death 1 on simian immunodeficiency virus-specific CD8 T cells during chronic infection but not after vaccination.J Virol. 2007;81(11):5819-5828.

10. Petrovas C, et al. SIV-specific CD8+ T cells express high levels of PD1 and cytokines but have impaired proliferative capacity in acute and chronic SIVmac251 infection. Blood. 2007;110(3):928-936.

11. Barber DL, et al. Restoring function in exhausted CD8 T cells during chronic viral infection. Nature. 2006;439(7077):682-687.

12. Maxmen A. David Masopust: tracking tapirs, T cells, and other David Masopusts. J Exp Med. 2009;206(12):2580-2581.
13. Finnefrock AC, et al. PD-1 blockade in rhesus macaques: impact on chronic infection and prophylactic vaccination. J Immunol. 2009; 182(2):980-987.

14. Velu V, et al. Enhancing SIV-specific immunity in vivo by PD-1 blockade. Nature. 2009;458(7235):206-210.

15. Dyavar Shetty R, et al. PD-1 blockade during chronic SIV infection reduces hyperimmune activation and microbial translocation in rhesus macaques. J Clin Invest. 2012;122(5):1712-1716.

16. Abel K, Alegria-Hartman MJ, Rothaeusler K, Marthas M, Miller CJ. The relationship between simian immunodeficiency virus RNA levels and the mRNA levels of alpha/beta interferons (IFNalpha/beta) and IFN-alpha/beta-inducible Mx in lymphoid tissues of rhesus macaques during acute and chronic infection. J Virol. 2002; 76(16):8433-8445.

17. Bosinger SE, Sodora DL, Silvestri G. Generalized immune activation and innate immune responses in simian immunodeficiency virus infection. Curr Opin HIV AIDS. 2011;6(5):411-418.

18. Estes JD, et al. Damaged intestinal epithelial integrity linked to microbial translocation in pathogenic simian immunodeficiency virus infections. PLoS Pathog. 2010;6(8):e1001052.

19. Huang X, et al. PD-1 expression by macrophages plays a pathologic role in altering microbial clearance and the innate inflammatory response to sepsis. Proc Natl Acad Sci U S A. 2009;106(15):6303-6308.

20. Said EA, et al. Programmed death-1-induced interleukin-10 production by monocytes impairs CD4+ $\mathrm{T}$ cell activation during HIV infection. Nat Med. 2010;16(4):452-459.

21. Geldmacher C, et al. Preferential infection and depletion of Mycobacterium tuberculosis-specific CD4 T cells after HIV-1 infection. J Exp Med. 2010;207(13):2869-2881. 